\title{
EQUIVARIANT HOMOTOPY THEORY
}

\author{
BY C. VASEEKARAN
}

Communicated by Glen E. Bredon, August 30, 1973

In this note we announce an obstruction theory for extending (continuous) equivariant maps defined on a certain class of $G$-spaces, where $G$ is a compact Lie group. The details of this work will be published elsewhere. Our results barely touch upon the attendant problem of providing techniques that would serve in practice for the computation of the obstruction groups. In general this last problem presents considerably greater difficulties than in the case of a finite group $G$, which has been treated fairly exhaustively in [1]. The author expresses his deep gratitude to Professor Glen E. Bredon in consultation with whom these results were obtained.

Let $G$ be a compact Lie group. If $H$ is a closed subgroup of $G$, a closed $G$-stem of type $(H)$ and equivariant dimension $n$ is defined to be a $G$ space which is equivariantly homeomorphic to $B^{n} \times G / H$, where $B^{n}$ is the standard $n$-cell, $G / H$ is the homogeneous $G$-space consisting of the left cosets of $H$ in $G$, and the action of $G$ is the product of the trivial action on $B^{n}$ and the usual action on $G / H$. A Hausdorff $G$-space $K$ is said to be a $G$-complex if it is filtered by an ascending sequence of closed invariant subspaces $K^{n}$, whose union is $K$, such that $K^{-1}=\varnothing$ and, for each $n$, $K^{n}$ is obtained from $K^{n-1}$ by attaching any number of $n$-stems by equivariant maps defined on the boundaries $S^{n-1} \times G / H$ of the standard $n$-stems $B^{n} \times G / H$. A $G$-complex $K$ is also required to have the topology coherent with the sequence of subspaces $K^{n}$. The least integer $n$ such that $K^{n}=K$ is called the equivariant dimension of $K$ and is denoted by $\operatorname{dim}_{G} K$. The class of $G$-complexes is the analogue in the equiyariant category of $\mathrm{CW}$-complexes in the topological category. (See [3] for the definition and some of the properties of a CW-complex.) When $G$ is a finite group $G$-complexes have been defined by Bredon in [1]. Our notion is derived from his and we extend his techniques to the more general case.

Matumoto has defined in [2] what he calls a $G$-CW-complex. His definition is equivalent to that of a $G$-complex $K$ whose orbit space $K / G$ is a locally finite CW-complex. He has also indicated in [2] a proof of the important result that a differentiable $G$-manifold is a $G$-CW-complex

AMS (MOS) subject classifications (1970). Primary 55B25, 57E10. 
(and, hence, also a $G$-complex) for any compact Lie group $G$. Matumoto has obtained some of our results that do not involve equivariant cohomology theory in the setting of $G$-CW-complexes. Our definition allows somewhat more freedom which is exploited, for example, in our representability theorem.

The first part of our results concerns the elements of equivariant homotopy theory. We prove equivariant versions of the homotopy extension theorem, the cellular approximation theorem and the Whitehead theorem for $G$-complexes. The main device is the application of Bredon's rule [1] that the equivariant maps from $G / H$ to a $G$-space $Y$ are in one-one correspondence with the points of $Y^{H}$, the subset of $Y$ consisting of all points which are left fixed by the action of every element in the subgroup $H$.

A $G$-complex $K$ is said to be 0-connected if it has a basepoint and the fixed-point set $K^{H}$ is connected for every closed subgroup $H$ of $G$.

THEOREM 1. A (contravariant) equivariant homotopy functor defined on the class of 0-connected G-complexes is representable, the classifying $G$-space itself being a G-complex.

The category of canonical orbits $\mathcal{O}_{G}$ is defined to be the category whose objects are the homogeneous spaces $G / H$ and whose morphisms are the equivariant homotopy classes of equivariant maps. A coefficient system is a contravariant functor from $\mathcal{O}_{G}$ to the category of abelian groups. For each coefficient system $M$ we define the equivariant cohomology groups $H_{G}^{n}(K, L ; M)$ of a pair of $G$-complexes $(K, L)$ with coefficients in $M$. These cohomology groups constitute an equivariant cohomology theory in the sense of Bredon [1]. Their construction proceeds via the construction of dual homology coefficient systems as in [1]. However in the case of a compact Lie group $G$, we must, so to speak, factor out the homology of the orbits in a $G$-complex to obtain the right dual homology theory. The groups $H_{G}^{n}(K, L ; M)$ are derived from cochain groups $C_{G}^{n}(K, L ; M)$, where the latter may be taken to be a group of functions defined on the $n$-stems of $K-L$.

Given a pair of $G$-complexes $(K, L)$, a $G$-space $Y$ such that $Y^{H}$ is nonempty, arcwise connected and $n$-simple for all $n$ and for every closed subgroup $H$ of $G$, and an equivariant map $f: K^{r} \cup L \rightarrow Y$, the problem of extending $f$ equivariantly to $K^{r+1} \cup L$ leads to an obstruction cochain $c_{f} \in C_{G}^{r+1}\left(K, L ; \varpi_{r}(Y)\right)$, where $\varpi_{r}(Y)$ is defined almost exactly as in [1]. The obstruction cochain is a cocycle and its cohomology class $\left[c_{f}\right]$ vanishes if, and only if, $f \mid K^{r-1} \cup L$ can be extended to $K^{r+1} \cup L$. Beyond the obstruction cochain the theory is formally the same as that in the topological case. Let $n$ be the least integer such that $\varpi_{r}(Y)=0$ for all $r<n$. Then there 
is defined a primary obstruction $\gamma^{n+1}(f) \in H_{G}^{n+1}\left(K, L ; \varpi_{n}(Y)\right)$ which depends only on the equivariant homotopy class of $f \mid L$.

THEOREM 2. Suppose that $H_{G}^{r+1}\left(K, L ; \varpi_{r}(Y)\right)=0$ for all

$$
n<r<\operatorname{dim}_{G}(K-L) .
$$

Then $f: L \rightarrow Y$ has an equivariant extension to $K$ if and only if $\gamma^{n+1}(f)=0$.

THEOREM 3. Suppose in addition that $H_{G}^{r}\left(K, L ; \varpi_{r}(Y)\right)=0$ for all $n<r \leqq \operatorname{dim}_{G}(K-L)$. Let $f: K \rightarrow Y$ be an equivariant map. Then there is a one-one correspondence between equivariant homotopy classes relative to $L$ of equivariant maps $g: K \rightarrow Y$ such that $g|L=f| L$ and the elements of $H_{G}^{n}\left(K, L ; \varpi_{n}(Y)\right)$.

We have formulated our theory in such a way that it is possible to restrict attention to $G$-complexes whose orbits all come from some limited subset of the set of all possible orbit-types. Thus the application of our theory leads to characteristic classes of certain $G$-complexes which are the primary obstructions to constructing global slices of prescribed types. In the case of a circle group $G$, Theorem 2 (slightly modified) tells us that the vanishing of this characteristic class is necessary and sufficient for the existence of a global slice.

Theorem 3 leads to criteria for the equivariant contractibility of a $G$-complex and to criteria for an equivariant map between $G$-complexes to be an equivariant homotopy equivalence. We also get from it a classification theorem for equivariant homotopy classes of maps into EilenbergMac Lane $G$-spaces which are defined by homotopy conditions as in [1], their existence being guaranteed by Theorem 1 .

\section{BIBLIOGRAPHY}

1. G. E. Bredon, Equivariant cohomology theories, Lecture Notes in Math., no. 34, Springer-Verlag, Berlin and New York, 1967. MR 35 \#4914.

2. T. Matumoto, Equivariant K-theory and Fredholm operators, J. Fac. Sci. Univ. Tokyo Sect. I A Math. 18 (1971), 109-125. MR 44 \#7538.

3. J. H. C. Whitehead, Combinatorial homotopy. I, Bull. Amer. Math. Soc. 55 (1949), 213-245. MR 11, 48.

Department of Mathematics, University of Ceylon, Colombo, Ceylon 